\title{
THE COMPLEX EQUILIBRIUM MEASURE OF A SYMMETRIC CONVEX SET IN $\mathbf{R}^{n}$
}

BY

\author{
ERIC BEDFORD AND B. A. TAYLOR ${ }^{1}$
}

\begin{abstract}
We give a formula for the measure on a convex symmetric set $K$ in $\mathbf{R}^{n}$ which is the Monge-Ampere operator applied to the extremal plurisubharmonic function $L_{K}$ for the convex set. The measure is concentrated on the set $K$ and is absolutely continuous with respect to Lebesgue measure with a density which behaves at the boundary like the reciprocal of the square root of the distance to the boundary. The precise asymptotic formula for $x \in K$ near a boundary point $x_{0}$ of $K$ is shown to be of the form $c\left(x_{0}\right) /[\operatorname{dist}(x, \partial K)]^{-1 / 2}$, where the constant $c\left(x_{0}\right)$ depends both on the curvature of $K$ at $x_{0}$ and on the global structure of $K$.
\end{abstract}

1. Introduction. Let us denote the family of plurisubharmonic (psh) functions on $\mathbf{C}^{n}$ of minimal growth by

$$
\mathcal{L}=\left\{v \text { psh on } \mathbf{C}^{n}, v(z) \leq \log (1+|z|)+O(1)\right\} .
$$

For $K$ a compact subset of $\mathbf{C}^{n}$ the extremal function $L_{K}^{*}$ for $K$ with logarithmic singularity at infinity is defined by setting

$$
L_{K}(z)=\sup \{v(z): v \in \mathcal{L}, v \leq 0 \text { on } K\}
$$

and

$$
L_{K}^{*}(z)=\limsup _{\zeta \rightarrow z} L_{K}(\zeta)
$$

(cf. Siciak [13] and Zaharjuta [17]). The function $L_{K}^{*}$ is in general not smooth on $\mathbf{C}^{n} \backslash K$ when $n>1$ and, in particular, it is not harmonic. It is a theorem of Siciak that either $L_{K}^{*} \equiv+\infty$, in which case the set $K$ is pluripolar, or else $L_{K}^{*} \in \mathcal{L}$. If $L_{K}$ is continuous on $\mathbf{C}^{n}$, then $L_{K} \equiv L_{K}^{*} \in \mathcal{L}$.

The extremal function $L_{K}^{*}$ satisfies the complex Monge-Ampere equation

$$
\left(d d^{c} L_{K}^{*}\right)^{n}=0
$$

in a generalized sense on $\mathbf{C}^{n} \backslash K$ [2, Corollary 9.4]. Thus, for nonpluripolar sets $K$,

$$
\lambda_{K}:=\left(d d^{c} L_{K}^{*}\right)^{n}
$$

is a positive Borel measure supported on $K$. It has total mass equal to $(2 \pi)^{n}$ (cf. [16]). We will call $\lambda_{K}$ the complex equilibrium measure for $K$.

Here we consider compact sets $K \subset \mathbf{R}^{n} \subset \mathbf{C}^{n}$. In this case $K$ is polynomially convex, and so $L_{K}>0$ on $\mathbf{C}^{n} \backslash K$. There are rather reasonable hypotheses on

Received by the editors May 7, 1985.

1980 Mathematics Subject Classification. Primary 32F05, 31C10.

Key words and phrases. Plurisubharmonic function, Monge-Ampere operator, extremal function.

${ }^{1}$ This research was supported in part by the NSF while the second author was visiting Indiana University. 
$K$ that will insure that $L_{K}$ is continuous on $\mathbf{C}^{n}$, such as:

$$
\begin{aligned}
& \text { for each } x_{0} \in \partial K, \text { there exists a real analytic curve } \\
& t \rightarrow \gamma(t) \in \mathbf{R}^{n},-1<t<+1 \text {, such that } \gamma(t) \in \operatorname{int}(K) \\
& \text { for }-1<t<0, \text { and } \gamma(0)=x_{0} .
\end{aligned}
$$

(See Plesniak [9] and Sadullaev [12].)

Our first result concerning the extremal measure for subsets $K \subset \mathbf{R}^{n}$ is that on compact subsets of the interior of $K, \lambda_{K}$ is equivalent to $n$-dimensional Lebesgue measure.

THEOREM 1.1. If $K \subset \mathbf{R}^{n}$ is compact, then there exist constants $0<c_{1}<$ $c_{2}<\infty$ such that

$$
\int_{E} c_{1} d x \leq \lambda_{K}(E) \leq \int_{E} \frac{c_{2} d x}{[\operatorname{dist}(x, \partial K)]^{n}}
$$

holds for any Borel set $E \subset \operatorname{int}(K)$. We can take

$$
c_{1}=2^{n}[\operatorname{diam}(K)]^{-n}, \quad c_{2}=(2 \sqrt{n})^{n} .
$$

In case $K \subset \mathbf{R}^{n}$ is convex and symmetric about the origin, the extremal function $L_{K}$ may be given in a relatively simple form. Starting with the case $n=1$, we note that a convex $K \subset \mathbf{R}$ is an interval. The extremal function for $K=[-1,+1] \subset \mathbf{C}$ is given by the familiar Green function

$$
L_{[-1,+1]}(z)=\psi(z):=\log \left|z+\sqrt{z^{2}-1}\right| .
$$

For convex $K \subset \mathbf{R}^{n}$, we have the support function

$$
\rho_{K}(\xi):=\sup _{x \in K} \xi \cdot x
$$

defined for $\xi \in \mathbf{R}^{n}$. It is a theorem of Lundin that the extremal function may be obtained as

$$
L_{K}(z)=\sup _{|\xi|=1, \xi \in \mathbf{R}^{n}} \psi\left(\frac{z \cdot \xi}{\rho_{K}(\xi)}\right)
$$

where $z \cdot \xi=z_{1} \xi_{1}+\cdots+z_{n} \xi_{n}$ is a projection to the complexification of the $\xi$-axis. Dividing by the support function $\rho_{K}(\xi)$ is a normalization so that $K$ projects to the interval $[-1,+1]$. The representation (1.4) was given by Lundin in [6].

For the case of convex symmetric sets, the extremal measure can also be computed in an explicit form.

THEOREM 1.2. Let $K \subset \mathbf{R}^{n}$ be a compact convex set, symmetric about the origin and with nonempty interior. Then $\lambda_{K}=n ! \cdot \lambda(x) d x$, where $\lambda(x)$ is the $n$ dimensional volume of the convex hull of $S_{x}\left(K^{*}\right)$, where $K^{*}$ is the convex set dual to $K$, and $S_{x}: \mathbf{R}^{n} \rightarrow \mathbf{R}^{n}$ is the map

$$
S_{x}(\eta)=\eta /\left(1-(x \cdot \eta)^{2}\right)^{1 / 2} .
$$

In case $S_{x}\left(K^{*}\right)$ is convex, then

$$
\lambda(x)=\frac{1}{n} \int_{|\xi|=1} \frac{d \sigma(\zeta)}{\left[\rho_{K}^{2}(\xi)-(x \cdot \xi)^{2}\right]^{n / 2}}, \quad x \in K
$$

( $d \sigma$ denotes surface area measure on the unit sphere). 
This theorem extends a result of Lundin [7], who has shown that when $K=\mathbf{B}^{n}$ is the unit ball in $\mathbf{R}^{n}$, then

$$
L_{\mathbf{B}^{n}}(z)=\sinh ^{-1}\left[\frac{1}{2}\left(|z|^{2}-1+\left|z^{2}-1\right|\right)\right]^{1 / 2}
$$

and that

$$
\lambda_{\mathbf{B}^{n}}=\frac{c_{n}}{\left(1-|x|^{2}\right)^{1 / 2}} d x, \quad \text { where } c_{n}=2^{n} \cdot \Gamma\left(\frac{n+1}{2}\right) \cdot \pi^{(n-1) / 2} .
$$

A less precise but more general result is as follows.

THEOREM 1.3. If $K \subset \mathbf{R}^{n}$ is compact and convex with nonempty interior and smooth boundary, then there are constants $0<c_{1}<c_{2}$ such that

$$
\frac{c_{1} d x}{[\operatorname{dist}(x, \partial K)]^{1 / 2}} \leq \lambda_{K} \leq \frac{c_{2} d x}{[\operatorname{dist}(x, \partial K)]^{1 / 2}} .
$$

We also compute the asymptotic behavior of $\lambda_{K}(x)$ at $\partial K$.

THEOREM 1.4. If $K$ is a smoothly bounded symmetric convex set with nonvanishing curvature, then there is a smooth function $c(x)$ on $\partial K$ such that

$$
\lambda_{K}(x) \approx c(\bar{x})|x-\bar{x}|^{-1 / 2}
$$

where $\bar{x} \in \partial K$ is the boundary point closest to $x$.

This theorem is proved in $\S 4$, where a geometric construction is given for the quantity $c(\bar{x})$. It turns out that $c(\bar{x})$ is given by the volume of a certain set in $\mathbf{R}^{n}$ (see Figure 3), which depends both on the curvature of $\partial K$ at $\bar{x}$ and a global geometric envelope, the "ellipsoidal hull", of $K$.

Our interest in knowing $\lambda_{K}$ more precisely in these specific cases arises from the connection between $L_{K}$ and some problems concerning polynomials. Theorem 1.3 gives a more concrete statement of the Leja polynomial condition in the case of a smoothly bounded convex set $K \subset \mathbf{R}^{n}$. The condition is as follows (cf. [14]). If $\mathcal{F}=\left\{P_{\alpha}: \alpha \in A\right\}$ is any family of polynomials and if

$$
S_{\mathcal{F}}=\left\{x \in K: \sup _{\alpha \in A}\left|p_{\alpha}(x)\right|=+\infty\right\}
$$

then the following are equivalent:

for any $\lambda>1$ there exists an open set $U \supset K$ and an $M<\infty$ such that for all polynomials in the family $₹$

and

$$
\sup _{U}\left|p_{\alpha}\right| \leq M \cdot \lambda^{\operatorname{deg}\left(p_{\alpha}\right)}
$$

the fine interior of $S_{\mp}$ has Lebesgue measure zero.

Here the "fine interior" is taken with respect to the plurifine topology induced from $\mathbf{C}^{n}$, cf. [3]. 
It was shown by Siciak [14] that if a measure $\mu$ satisfies the Leja polynomial condition, in the sense that (1.6) holds whenever $\mu\left(S_{\mathcal{F}}\right)=0$, then it also satisfies a version of the Bernstein-Markov condition:

for $0<s<+\infty, \lambda>1$, there exist an open set $U \supset K$, and a constant $C_{s}<\infty$ such that for any polynomial $p$,

$$
\sup _{U}|p| \leq C_{s} \cdot \lambda^{\operatorname{deg}(p)}\left[\int|p|^{s} d \mu\right]^{1 / s} .
$$

This inequality is used in polynomial approximation and analytic continuation. It follows from the results above that any measure $\mu$ with $\left.d x\right|_{K} \ll \mu$ will satisfy (1.7). There is evidence, however, that the measure $\lambda_{K}$ should be in some sense optimal in these problems. For instance, it is conjectured in [15] that $\lambda_{K}$ should reflect the asymptotic behavior of the extremal points of $K$.

2. Equivalence of $\lambda_{K}$ and Lebesgue measure. In this section we will prove a comparison result which is closely related to a theorem of Levenberg [5]. This will lead to a proof of Theorem 1.1.

We will denote by $P(\Omega)$ the space of all psh functions on the domain $\Omega$ in $\mathbf{C}^{n}$.

LEMMA 2.1. Let $u_{1}, u_{2} \in P(\Omega)$ be given locally bounded functions, and let $S \subset \Omega \cap \mathbf{R}^{n}$ be a closed set containing the supports of the Borel measures $\left(d d^{c} u_{1}\right)^{n}$ and $\left(d d^{c} u_{2}\right)^{n}$. If the sets $\left\{u_{1}=0\right\}$ and $\left\{u_{2}=0\right\}$ differ from $S$ by at most $a$ pluripolar set, and if $0 \leq u_{1} \leq u_{2}$ on $\Omega$, then $\left(d d^{c} u_{1}\right)^{n} \leq\left(d d^{c} u_{2}\right)^{n}$.

ProOF. Without loss of generality, we may assume that $B=\{|z|<1\}$ is a compact subset of $\Omega$. Let $u_{j}^{\varepsilon}=u_{j} * \chi_{\varepsilon}$ be smoothings decreasing to $u_{j}$ as $\varepsilon \downarrow 0$. Let $v_{j}$ denote the restriction of $u_{j}$ to $\partial B$. Similarly, let $v_{j}^{\varepsilon}$ denote the restriction of $u_{j}^{\varepsilon}+j \cdot \varepsilon$ to $\partial B$, so that $0<v_{1}^{\varepsilon}<v_{2}^{\varepsilon}$ on $\partial B$. Given a subset $S$ of the unit ball $B$ and a function $v$ on $\partial B$, let $\mathcal{F}(S, v)$ denote the family of all psh functions $w$ on the unit ball $B$ such that $w \leq 0$ on $S$, and $\limsup _{\zeta \rightarrow z} w(\zeta) \leq v(z)$ for all $z \in \partial B$. Let

$$
S_{\delta}^{\varepsilon}=\left\{x \in \mathbf{R}^{n} \cap \Omega: \operatorname{dist}(x, \partial \Omega) \geq \varepsilon \text { and } \operatorname{dist}(x, S) \leq \delta\right\}
$$

and let

$$
U_{\delta, j}^{\varepsilon}(z):=\sup \left\{w(z): w \in \mathcal{F}\left(S_{\delta}^{\varepsilon}, v_{j}^{\varepsilon}\right)\right\}, \quad z \in B .
$$

The set $S_{j}^{\varepsilon}$ is easily seen to be regular, so $U_{\delta, j}^{\varepsilon}$ is psh and continuous on the closure $\bar{B}$ of $B$. It therefore follows from a theorem of Levenberg [5] that

$$
\left(d d^{c} U_{\delta, 1}^{\varepsilon}\right)^{n} \leq\left(d d^{c} U_{\delta, 2}^{\varepsilon}\right)^{n}
$$

As $\delta \downarrow 0$, the functions $U_{\delta, j}^{\varepsilon}$ increase almost everywhere to the functions $\left[U_{S, j}^{\varepsilon}\right]^{*}$, the uppersemicontinuous regularizations of the envelope functions for the families $\mathcal{F}\left(S, v_{j}^{\varepsilon}\right)$. Because the operator $\left(d d^{c}\right)^{n}$ is continuous on monotone limits of locally bounded psh functions [2], we can pass to the limit in the last inequality to obtain

$$
\left(d d^{c} U_{S, 1}^{\varepsilon}\right)^{n} \leq\left(d d^{c} U_{S, 2}^{\varepsilon}\right)^{n} .
$$

Now, let $\varepsilon \downarrow 0$. The functions $U_{S, j}^{\varepsilon}$ clearly decrease. And, they decrease to the uppersemicontinuous regularization of the envelope function of the family $\mathcal{F}\left(S, v_{j}\right)$, $U_{S, j}^{*}$. Thus, we have

$$
\left(d d^{c} U_{S, 1}^{*}\right)^{n} \leq\left(d d^{c} U_{S, 2}^{*}\right)^{n} .
$$


But, it is evident that $U_{S, j}^{*} \geq u_{j}$. On the other hand, we have $u_{j} \geq U_{S, j}^{*}$ by the domination principle (see [2, Corollary 4.5]). Thus, $u_{j}=U_{S, j}$ which proves the lemma.

Proposition 2.2. Let $E_{1}, \ldots, E_{n}$ be compact subsets of $\mathbf{C}$. Then

$$
\lambda_{E_{1} \times \cdots \times E_{n}}=\lambda_{E_{1}} \otimes \cdots \otimes \lambda_{E_{n}} \text {. }
$$

PROOF. By a result of Siciak [13] we know that

$$
L_{E_{1} \times \cdots \times E_{n}}^{*}\left(z_{1}, \ldots, z_{n}\right)=\max _{1 \leq j \leq n} L_{E_{j}}^{*}\left(z_{j}\right) .
$$

Let us assume that $E_{j}$ is a Jordan domain in $\mathbf{C}$ with real analytic boundary. Then $L_{E_{j}}$ may be extended from $\mathbf{C} \backslash E_{j}$ to a function $\tilde{L}_{j}$ which is harmonic in a neighborhood of $\partial E_{j}$. Thus we may write

$$
L_{E_{1} \times \cdots \times E_{n}}^{*}\left(z_{1}, \ldots, z_{n}\right)=\max _{1 \leq j \leq n}\left\{\tilde{L}_{1}, \ldots, \tilde{L}_{n}, 0\right\} .
$$

However,

$$
\left(d d^{c} L_{E_{1} \times \cdots \times E_{n}}\right)^{n}=\left.d^{c} \tilde{L}_{1} \wedge \cdots \wedge d^{c} \tilde{L}_{n}\right|_{M}
$$

where $M=\left\{\tilde{L}_{1}=\cdots=\tilde{L}_{n}=0\right\}$ is given the orientation of $d \tilde{L}_{1} \wedge \cdots \wedge d \tilde{L}_{n}$ (see the remark on p. 7 of $[\mathbf{1}])$. Since

$$
d^{c} \tilde{E}_{j}=\frac{\partial}{\partial n_{j}}\left(\tilde{L}_{j}\right) d \sigma_{j}
$$

where $d \sigma_{j}$ is the arclength measure of $\partial E_{j}$ and $d n_{j}$ is the outward normal, we see that $d^{c} \tilde{L}_{j}$ may be naturally identified with $\lambda_{E_{j}}$. This proves the proposition in the smooth case.

For the general case, we take smooth $E_{j}^{\varepsilon}$,

$$
E_{j} \subset E_{j}^{\varepsilon} \subset\left\{z \in \mathbf{C}: \operatorname{dist}\left(z, E_{j}\right) \leq \varepsilon\right\} .
$$

If we take $E_{j}^{\varepsilon} \subset E_{j}^{\delta}$ for $\varepsilon \leq \delta$, then $L_{E_{1}^{\varepsilon} \times \cdots \times E_{n}^{\varepsilon}}^{*}$ increases a.e. to $L_{E_{1} \times \cdots \times E_{n}}$. Thus, by the convergence theorem of [2],

$$
\lim _{\varepsilon \rightarrow 0} \lambda_{E_{1}^{\varepsilon} \times \cdots \times E_{n}^{\varepsilon}}=\lambda_{E_{1} \times \cdots \times E_{n}}
$$

and the proposition is proved.

In the case of the interval $[-1,+1]$, it is well known that

$$
\lambda_{[-1,+1]}=(2 \cdot d x) /\left(1-x^{2}\right)^{1 / 2},
$$

and so a product of intervals,

$$
E=\left\{-\delta \leq x_{1} \leq \delta, \ldots,-\delta \leq x_{n} \leq \delta\right\}
$$

has extremal measure

$$
\lambda_{E}=\frac{2^{n} \cdot d x_{1} \cdots d x_{n}}{\delta^{n} \cdot\left[1-\left(x_{1} / \delta\right)^{2}\right]^{1 / 2} \cdots\left[1-\left(x_{n} / \delta\right)^{2}\right]^{1 / 2}} .
$$

PROOF OF THEOREM 1.1. If $r$ is the diameter of $K$, then without loss of generality we may assume that $K \subset(r \cdot I)^{n} \subset \mathbf{R}^{n}$ where $r \cdot I=[-r,+r]$. Thus, $L_{K}^{*} \geq L_{(r \cdot I)^{n}}^{*}$. If $x_{0} \in \operatorname{int}(K)$, then $\operatorname{dist}\left(x_{0}, \partial K\right)=\eta>0$. Without loss of 
generality, we can assume that $x_{0}=0$. Let $\Omega=\left\{z \in \mathbf{C}^{n}:|z|<\eta\right\}$ and $S=\Omega \cap \mathbf{R}^{n}$. It follows that $S=\left\{L_{K}^{*}=0\right\} \cap \Omega=\left\{L_{(r \cdot I)^{n}}^{*}=0\right\} \cap \Omega$ and so by Lemma 2.1,

$$
\begin{aligned}
\lambda_{K} \mid S & \geq\left.\frac{2^{n} \cdot d x_{1} \cdots d x_{n}}{r^{n} \cdot\left[1-\left(x_{1} / r\right)^{2}\right]^{1 / 2} \cdots\left[1-\left(x_{n} / r\right)^{2}\right]^{1 / 2}}\right|_{S} \\
& \geq\left.\frac{2^{n} \cdot d x_{1} \cdots d x_{n}}{r^{n}}\right|_{S} .
\end{aligned}
$$

For the other inequality, we note that $\left\{x:-\eta / \sqrt{n} \leq x_{j} \leq+\eta / \sqrt{n}\right\} \subset \operatorname{int}(K)$, so again by Lemma 2.1

$$
\frac{[2 \sqrt{n}]^{n} \cdot d x_{1} \cdots d x_{n}}{(\operatorname{dist}(x, \partial K))^{n / 2}} \geq \lambda_{K} \mid \operatorname{int}(K) .
$$

This completes the proof.

PROOF OF THEOREM 1.3. First let us assume that $\partial K$ is real analytic. Thus, it is pluripolar and $\lambda_{K}(\partial K)=0$. Since $\partial K$ is $C^{2}$, we may touch any $x_{0} \in K$ by an internally tangent ball of fixed radius $r$. Thus, they may use an internally tangent ball and an externally tangent cube to obtain (1.5) as in Theorem 1.1, because there is no mass on $\partial K$.

If $\partial K$ is merely $C^{2}$, we may consider a sequence $\left\{K_{j}\right\}$ of convex, symmetric domains with real analytic boundary such that $\partial K_{j}$ approaches $\partial K$ in $C^{2}$. Thus, we obtain (1.5) for each $\lambda_{K_{j}}$. Further, the constants $c_{1}$ and $c_{2}$ depend only on the diameter of $K_{j}$ and the radius of an internally tangent ball. Thus, we may be chosen independent of $j$. It follows from the dominated convergence theorem, then, that $\lambda_{K}$ puts no mass on $\partial K$, so that (1.5) holds.

3. Representation of $\left(d d^{c} L_{K}\right)^{n}$. In this section we will use Lundin's representation (1.4) of $L_{K}$ and give the basic form of $\left(d d^{c} L_{K}\right)^{n}$ for convex sets in $\mathbf{R}^{n}$. We first give a geometrical construction of an auxillary function that will be used to describe $\left(d d^{c} L_{K}\right)^{n}$.

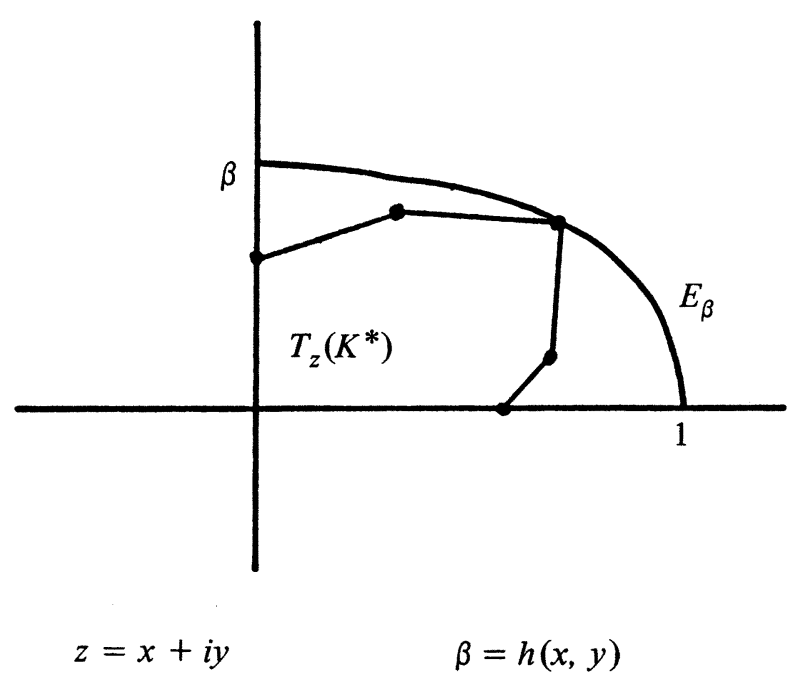


For $\beta>0$, let $E_{\beta}$ denote the ellipse

$$
E_{\beta}: \quad s^{2}+t^{2} / \beta^{2}=1 .
$$

Let $K^{*}$ denote the dual convex set to $K$. That is,

$$
K^{*}=\left\{\eta \in \mathbf{R}^{n}: x \cdot \eta \leq 1 \text { for all } x \in K\right\} .
$$

It is readily checked that when $K$ is convex and symmetric about the origin, so is $K^{*}$ and

$$
K^{*}=\left\{\eta=r \cdot \alpha \in \mathbf{R}^{n}:|\alpha|=1, r \leq 1 / \rho(\alpha)\right\} .
$$

We will use the function $h=h_{K}$ defined for $z=x+i \cdot y$ with $x \in \operatorname{int}(K), y \in \mathbf{R}^{n}$ by

$$
h_{K}(z)=h(x, y)=\inf \left\{\beta: T_{z}\left(K^{*}\right) \subset E_{\beta}\right\}
$$

(see Figure 1) where $T_{z}$ is the linear map from $\mathbf{R}^{n}$ to $\mathbf{R}^{2}$ defined by $\zeta=s+i \cdot t=$ $T_{z}(\xi)=z \cdot \xi$, or, more precisely by

$$
\left[\begin{array}{c}
s \\
t
\end{array}\right]=T_{z}(\xi)=\left[\begin{array}{ccc}
x_{1} & & x_{n} \\
& \ldots & \\
y_{1} & & y_{n}
\end{array}\right] \cdot\left[\begin{array}{c}
\xi_{1} \\
\vdots \\
\xi_{n}
\end{array}\right] .
$$

The function $h(x, y)=h_{K}(x, y)$ has the following properties.

PROPOSITION 3.1. If $K^{*}$ is the dual convex set to the compact convex set $K$, and if $K^{*}$ is compact, then for $h(x, y)$ defined in (3.2) we have

(i) $h(x, y)$ is continuous for $x \in \operatorname{int}(K)$ and $y \in \mathbf{R}^{n}$;

(ii) $h(x, \lambda \cdot y)=\lambda \cdot h(x, y)$ for $\lambda>0$;

(iii) $y \rightarrow h(x, y)$ is convex on $R^{n}$ for each fixed $x \in \operatorname{int}(K)$;

(iv) $y \rightarrow h(x, y)$ is the support function of the convex hull of the set, $S_{x}\left(K^{*}\right)$, where $S_{x}$ is the (nonlinear) transformation of $\mathbf{R}^{n}$ defined by

$$
S_{x}(\xi)=\xi /\left(1-(x \cdot \xi)^{2}\right)^{1 / 2} .
$$

ProOF. First, note that $h$ is defined and finite when $x \in \operatorname{int}(K)$ because then $\xi \cdot x<1$ for all $\xi \in K^{*}$, so the set $T_{z}\left(K^{*}\right)$ is contained in $E_{\beta}$ for some $\beta>0$. That $h$ is continuous is clear because $K^{*}$ is compact. Assertion (ii) follows from the obvious scaling property

$$
T_{x+i \cdot \lambda \cdot y}\left(K^{*}\right) \subset E_{\beta} \quad \text { iff } \quad T_{x+i \cdot y}\left(K^{*}\right) \subset E_{\beta / \lambda} .
$$

Both assertions also follow directly from (iv), as does assertion (iii). We will therefore prove assertion (iv).

The number $\beta=h(x, y)$ is defined as the smallest number $\beta \geq 0$ such that

$$
(x \cdot \xi)^{2}+(y \cdot \xi)^{2} / \beta^{2} \leq 1, \quad \xi \in K^{*},
$$

with equality holding for at least one $\xi$. Solving the inequality for $\beta$ yields

$$
\beta \geq y \cdot \xi /\left(1-[x \cdot \xi]^{2}\right)^{1 / 2}=y \cdot S_{x}(\xi), \quad \xi \in K^{*},
$$

with equality for at least one $\xi$. In other words, $h(x, y)$ is the maximum of the right-hand side of (3.3) over $K^{*}$, which is exactly assertion (iv). This completes the proof of the proposition. 


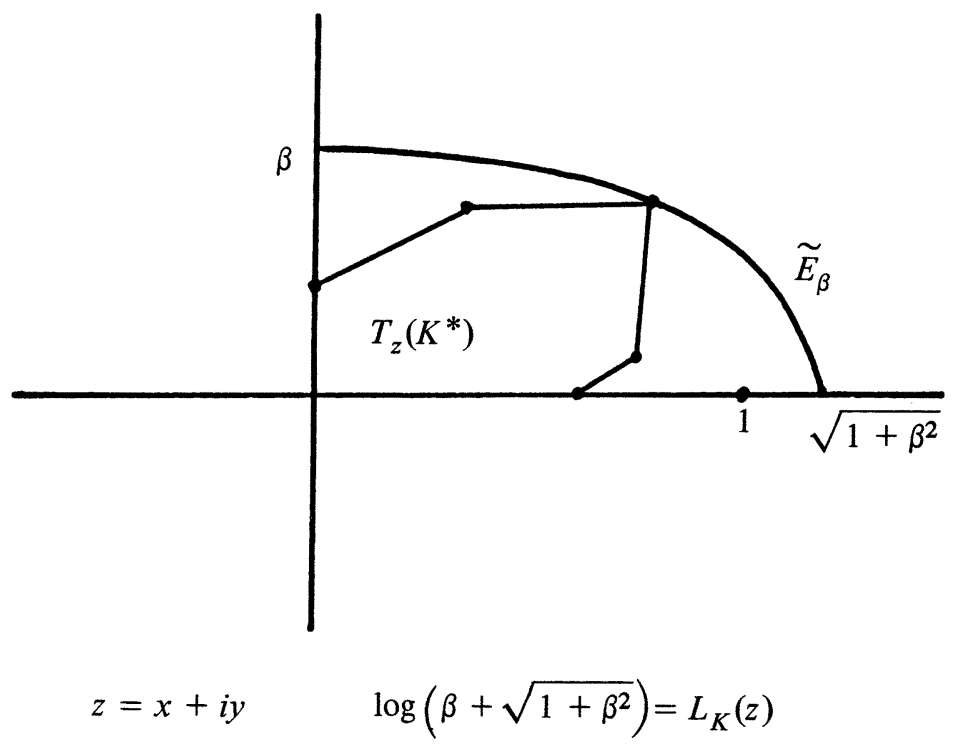

FiguRE 2

For $f$ a convex function of $y \in \mathbf{R}^{n}$, let $M(f)$ denote the real Monge-Ampere operator applied to $f$. That is, if $f$ is smooth, then $M(f)$ is the determinant of the Hessian of $f$,

$$
M(f)=\operatorname{det}\left[\partial^{2} f / \partial y_{j} \partial y_{k}\right]
$$

The operator extends by continuity to be defined as a nonnegative Borel measure on the class of convex functions. The measure of a Borel set $E$ is the volume of the set of all direction vectors $\lambda$ of hyperplanes, $\lambda \cdot y+$ const., which support the graph of $f$ at a point $x \in E$. See e.g. [11].

We will use two facts about this operator. First, if the convex function $f(y)$ defined on $\mathbf{R}^{n}$ is thought of as a psh function on $\mathbf{C}^{n}$, then we have the formula

$$
\left(d d^{c} f\right)^{n}=n ! \cdot M(f) \otimes d x .
$$

Second, if $f$ is the function $y \rightarrow h(x, y)$, then because $h$ is homogeneous of degree 1 , $M(f)$ must be supported at the origin. Further, this set of direction vectors which support the graph of the function is clearly the convex hull of the set $S_{x}\left(K^{*}\right)$. Thus, if $x_{0}$ is a fixed point of $\operatorname{int}(K)$, then

$$
\left(d d^{c} h\left(x_{0}, y\right)\right)^{n}=n ! \cdot \operatorname{vol}\left\{\operatorname{ch} S_{x}\left(K^{*}\right)\right\} \cdot d x .
$$

We next show the relation between the function $h=h_{K}$ and the extremal function $L_{K}$.

THEOREM 3.2. Let $K$ be a convex compact symmetric set in $\mathbf{R}^{n}$ with $0 \in$ $\operatorname{int}(K)$. Then

$$
\lim _{\varepsilon \rightarrow 0^{+}} \frac{L_{K}(x+i \cdot \varepsilon \cdot y)}{\varepsilon}=h_{K}(x, y)
$$

uniformly on compact subsets of $\operatorname{int}(K) \oplus i \mathbf{R}^{n} \subset \mathbf{C}^{n}$. In particular, if $x_{0} \in \operatorname{int}(K)$ and $\varepsilon>0$ is given, there exists $\delta>0$ such that, for all $\left|x-x_{0}\right| \leq \delta,|y| \leq \delta$,

$$
h_{K}\left(x_{0}, y\right)-\varepsilon \cdot|y| \leq L_{K}\left(x_{0}+i \cdot y\right) \leq h_{K}\left(x_{0}, y\right)+\varepsilon \cdot|y| \text {. }
$$


PROOF. Recall that the level sets of the function

$$
g(\varsigma):=\left|\varsigma+\sqrt{\varsigma^{2}-1}\right|
$$

are the confocal ellipses

$$
\tilde{E}_{\beta}: \quad s^{2} /\left(1+\beta^{2}\right)+t^{2} / \beta^{2}=1, \quad \varsigma=s+i \cdot t, \beta>0,
$$

and $g=\beta+\sqrt{1+\beta^{2}}$ on $\tilde{E}_{\beta}$. According to Lundin's formula, (1.4), choosing $\beta=\beta(z)$ such that $L_{K}(z)=\log \left[\beta+\sqrt{1+\beta^{2}}\right]$ is the same as

$$
\beta+\sqrt{1+\beta^{2}}=\sup \{g(z \cdot \xi / \rho(\xi)):|\xi|=1\} .
$$

Equivalently, $\beta=\beta(z)$ is defined by

$$
\beta(z)=\inf \left\{\beta: T_{z}\left(K^{*}\right) \subset \tilde{E}_{\beta}\right\}
$$

(see Figure 2). Note also that $d \beta / d g=1$ at $g=1, \beta=0$. The limit in (3.5) is therefore equivalent to calculating

$$
\lim _{\varepsilon \rightarrow 0^{+}} \frac{\beta(x+i \varepsilon y)}{\varepsilon} .
$$

We will calculate this limit by comparing it with the function $h$ defined in terms of the ellipses $E_{\beta}$ in (3.2). Notice that $E_{\beta} \subset \tilde{E}_{\beta}$ for all $\beta>0$ so that for all $z=x+i y \in C^{n}$

$$
h(x, y) \geq \beta(x+i y)
$$

On the other hand, if $x \in \operatorname{int}(K)$ then for sufficiently small $y \in \mathbf{R}^{n}$, the first components, $s=x \cdot \xi$, of $T_{z}\left(K^{*}\right)$ are contained in $[-r,+r]$ for some $r<1$. However, if $\lambda \geq 1$, then $\lambda \cdot E_{\beta} \cap\{(s, t):|s| \leq r\} \subset E_{a \cdot \beta}$ for $a \geq\left[\left(\lambda^{2}-r^{2}\right) /\left(1-r^{2}\right)\right]^{1 / 2}$. Further, $\tilde{E}_{\beta} \subset \sqrt{1+\beta^{2}} \cdot E_{\beta}$. Consequently, if $T_{z}\left(K^{*}\right) \subset \tilde{E}_{\beta}$, then

$$
T_{z}\left(K^{*}\right)=T_{z}\left(K^{*}\right) \cap\{(s, t):|s| \leq r\} \subset E_{a \cdot \beta}
$$

for $a=\left[1+\beta^{2} /\left(1-r^{2}\right)\right]^{1 / 2} \approx 1+$ const. $\cdot \beta^{2}$. That is,

$$
\beta(x+i y) \geq h(x, y) \cdot\left[1+\text { const } \cdot \beta^{2}\right]^{-1 / 2} .
$$

From (3.9), (3.10), and the definition (3.2) of $h$, it therefore follows that the limit in (3.5) is equal to $h(x, y)$. All the set inclusions used above vary continuously with $x$, so it is easy to see that the limit is uniform on compact subsets of $\operatorname{int}(K) \oplus i \mathbf{R}^{n}$. This completes the proof.

PROOF OF THEOREM 1.2. First we note that it may be argued as in the proof of Theorem 1.3 to see that $\lambda_{K}$ puts no mass on $\partial K$. Now the formula for $\lambda(x)$ follows directly from formula (3.4), inequality (3.6) of Theorem 3.2, and the comparison theorem of $\S 2$. The integral formula may be seen as follows. The set $S_{x}\left(K^{*}\right)$ is given by $\left\{\eta=r \cdot \xi: 0 \leq r \leq\left(\left[\rho_{K}(\xi)\right]^{2}-[x \cdot \xi]^{2}\right)^{-1 / 2}\right\}$, and so the integral formula gives the volume of this region in polar coordinates.

REMARK. We note that $\operatorname{ch} S_{x}\left(K^{*}\right)$ is the dual of the set

$$
\left\{y \in \mathbf{R}^{n}:(y \cdot \xi)^{2}+(x \cdot \xi)^{2} \leq \rho_{K}^{2}(\xi) \text { for all } \xi \in \mathbf{R}^{n}\right\} .
$$

Since the total mass of $\lambda_{K}$ is independent of $K$, it follows that the constant

$$
c_{n}=\int \operatorname{vol}\left(\operatorname{ch} S_{x}\left(K^{*}\right)\right) d x
$$

is the same for all symmetric convex sets $K \subset \mathbf{R}^{n} .\left[c_{n}=(2 \pi)^{n} / n !\right.$.] It would be interesting to know if there is a purely geometrical proof of this fact. 
4. Asymptotic behavior of $\lambda_{K}$. Let $K \subset \mathbf{R}^{n}$ be smoothly bounded, convex, and symmetric. We will discuss the asymptotic behavior of $\lambda(x)$, and, in the process, prove Theorem 1.4. By Theorem 1.2, we will need to estimate the asymptotic behavior of the volume of the convex hull of $S_{x}\left(K^{*}\right)$ as $x$ approaches $x_{0} \in \partial K$.

First we establish some geometric notation. Let $\eta_{0}$ be a normal vector to $\partial K$ at $x_{0}$, normalized so that $\eta_{0} \cdot x_{0}=1$. Any ellipsoid symmetric about $0 \in \mathbf{R}^{n}$ and tangent to $\partial K$ at $x_{0}$ has the form

$$
\left(\eta_{0} \cdot x\right)^{2}+R(x) \leq 1
$$

where $R(x)$ is a positive semidefinite quadratic form on $\mathbf{R}^{n}$ such that $R\left(x_{0}\right)=0$. By abuse of notation, $R$ may be identified with a positive semidefinite quadratic form on the hypersurface $x_{0}^{\perp}$ orthogonal to $x_{0}$.

We define the ellipsoidal hull of $K$ at $x_{0}$, written $\mathrm{EH}\left(K, x_{0}\right)$, as the intersection of all the ellipsoids of the form (4.1) which contain $K$. Similarly, we define the ellipsoidal core of $K$ at $x_{0}$, written $\operatorname{EC}\left(K, x_{0}\right)$, as the union of all of the ellipsoids of the form (4.1) which are contained in $K$. The ellipsoidal hull and core are related as follows, which is proved in [4].

PROPOSITION 4.1. If $K$ and $K^{*}$ are both smoothly bounded, then $\operatorname{EC}\left(K, x_{0}\right)^{*}=$ $\mathrm{EH}\left(K^{*}, \eta_{0}\right)$.

Next, we relate the ellipsoidal hull to the mapping

$$
S_{x_{0}}(\eta)=\eta \cdot\left(1-\left(x_{0} \cdot \eta\right)^{2}\right)^{-1 / 2}
$$

LEMMA 4.2. $\operatorname{ch} S_{x_{0}}\left(K^{*}\right)=S_{x_{0}}\left\{\mathrm{EH}\left(K^{*}, \eta_{0}\right)\right\}$.

ProOF. We note that $\xi=S_{x_{0}}(\eta)$ carries the degenerate ellipsoid $\left(\xi_{0} \cdot \eta\right)^{2}+$ $\left(x_{0} \cdot \eta\right)^{2} \leq 1$ to the "strip" $\left|\xi_{0} \cdot \xi\right| \leq 1$. If $\xi_{0} \perp \eta_{0}$, then these degenerate ellipsoids are tangent to $K^{*}$ at $\eta_{0}$, and thus the set of these ellipsoids with $\xi_{0} \perp \eta_{0}$ generates the ellipsoidal hull. Thus,

$$
S_{x_{0}}\left\{\operatorname{EH}\left(K^{*}, \eta_{0}\right)\right\}=\bigcap\left\{\left|\xi_{0} \cdot \xi\right| \leq 1\right\}
$$

where the $\xi_{0}$ are chosen so that $\xi_{0} \perp \eta_{0}$ and the corresponding degenerate ellipsoid contains $K^{*}$. Thus, $\left\{\left|\xi_{0} \cdot \xi\right| \leq 1\right\} \supset S_{x_{0}}\left(K^{*}\right)$, and so $\operatorname{ch} S_{x_{0}}\left(K^{*}\right) \subset$ $S_{x_{0}}\left\{\mathrm{EH}\left(K^{*}, \eta_{0}\right)\right\}$.

On the other hand, $x_{0} \cdot \eta_{0}=1$, and so $S_{x_{0}}\left(K^{*}\right)$ contains the line $\left\{t \eta_{0}: t \in \mathbf{R}\right\}$. It follows that $\operatorname{ch} S_{x_{0}}\left(K^{*}\right)$ is an intersection of half-spaces of the form $\left\{\xi_{0} \cdot \xi \leq 1\right\}$ with $\xi_{0} \cdot \eta_{0}=0$. This proves the lemma.

LEMMA 4.3. For $x=t x_{0}, 0<t<1$, let us set

$$
\omega_{t}=\left(\operatorname{ch} S_{x}\left(K^{*}\right)\right) \cap\left(\eta_{0}^{\perp}\right) .
$$

Then $\omega_{t}$ increases to $S_{x_{0}}\left(\mathrm{EH}\left(K^{*}, \eta_{0}\right)\right) \cap\left(\eta_{0}^{\perp}\right)$ as $t$ increases to 1 .

PROOF. This follows from Lemma 4.2 since $\omega_{t}$ is clearly increasing in $t$.

Now we extend $\eta_{0}$ to an orthogonal set of coordinate axes, $\left(\eta_{0}, \eta_{1}, \ldots, \eta_{n-1}\right)$ such that $\eta_{1}, \ldots, \eta_{n-1}$ are the principal curvature directions of $\partial K$ at $x_{0}$, and we let $\kappa_{1}, \ldots, \kappa_{n-1}$ be the principal curvatures of $\partial K$ at $x_{0}$. Let $\left(\xi_{0}, \ldots, \xi_{n-1}\right)$ denote coordinates with respect to these new axes, and let $W_{t}: \mathbf{R}^{n} \rightarrow \mathbf{R}^{n}$ be the linear transformation given by

$$
W_{t}(\xi)=\left(\left(1-t^{2}\right)^{1 / 2} \cdot \xi_{0}, \xi_{1}, \ldots, \xi_{n-1}\right)
$$


Let $Q$ be the quadratic form on $\mathbf{R}^{n}$ which in $\xi$-coordinates is

and define the ellipsoid

$$
Q(\eta)=\sum_{j=1}^{n-1} \frac{\xi_{j}^{2}}{\kappa_{j}}
$$

$$
E=\left\{\eta \in \mathbf{R}^{n}: \xi_{0}^{2}+Q(\xi) \leq 1\right\}
$$

LEMMA 4.4.

$$
\lim _{t \rightarrow 1} W_{t}\left[S_{t x_{0}}\left(K^{*}\right) \cap\left\{\mathbf{R}^{n} \backslash \eta_{0}^{\perp}\right\}\right]=E .
$$

PROOF. Since $\pm \eta_{0}$ are the only points of $\partial K^{*}$ which get mapped to infinity under $S_{x_{0}}$, it follows that the left-hand side of (4.3) is determined only by a neighborhood of $\pm \eta_{0}$ in $\partial K^{*}$. That is, if $\tilde{\eta} \in \partial K^{*}$ and $\tilde{\eta} \neq \pm \eta_{0}$, then $\lim _{t \rightarrow 1} W_{t} S_{t x_{0}}(\tilde{\eta}) \in$ $\eta_{0}^{\perp}$. Thus, as $t \rightarrow 1$, we must consider smaller and smaller neighborhoods in order to find the left-hand side of (4.3).

Consider the ellipsoid which is symmetric about 0 and which is tangential to $\partial K^{*}$ at $\pm \eta_{0}$. The normal to $K^{*}$ at $\eta_{0}$ is $x_{0}$, so this ellipsoid has the form $\left(x_{0} \cdot \eta\right)^{2}+R(\eta) \leq$ 1 , as in (4.1). Since $\eta_{0} \cdot x_{0}=1$ and the curvatures of $\partial K^{*}$ at $\eta_{0}$ are $\kappa_{1}^{-1}, \ldots, \kappa_{n-1}^{-1}$, it follows that this ellipsoid coincides with

$$
\tilde{E}=\left\{\eta \in \mathbf{R}^{n}:\left(\eta \cdot x_{0}\right)^{2}+Q(\eta) \leq 1\right\}
$$

Without loss of generality, we may assume that $\partial K^{*}$ coincides with $\partial \tilde{E}$ in a small neighborhood of $\pm \eta_{0}$. Now it suffices to show that

$$
\lim _{t \rightarrow 1} W_{t} S_{t x_{0}}(\tilde{E})=E \text {. }
$$

To see this we consider first the ellipsoid

$$
\tilde{E}_{c}=\left\{\eta:\left(\eta \cdot x_{0}\right)^{2} / c^{2}+Q(\eta) \leq 1\right\}
$$

We see that

$$
S_{t x_{0}}^{-1}\left(E_{c}\right)=\left\{\eta:\left(\eta \cdot x_{0}\right)^{2} / c^{2}+Q(\eta) \leq 1-t^{2}\left(\eta \cdot x_{0}\right)^{2}\right\}
$$

is equal to $\tilde{E}$ if and only if $c^{2}=\left(1-t^{2}\right)^{-1}$. Thus

$$
W_{t}\left[S_{t x_{0}}(\tilde{E})\right]=W_{t}\left[\tilde{E}_{c}\right]
$$

with $c^{2}=\left(1-t^{2}\right)^{-1}$, and so we have, with $\eta=\xi_{0} \eta_{0}+\cdots+\xi_{n-1} \eta_{n-1}$,

$$
W_{t}\left[S_{t x_{0}}(\tilde{E})\right]=\left\{\eta \in \mathbf{R}^{n}:\left(1-t^{2}\right)\left[\frac{\xi_{0}}{\sqrt{1-t^{2}}}+\sum_{j=1}^{n-1} \xi_{j}\left(\eta_{j} \cdot x_{0}\right)\right]^{2}+Q(\eta) \leq 1\right\}
$$

Taking the limit as $t \rightarrow 1$, we obtain $E$.

Now we define $\omega=\left(\eta_{0}^{\frac{1}{1}}\right) \cap \operatorname{EH}\left(K^{*}, \eta_{0}\right), \Omega=\operatorname{ch}(\omega \cup E)$ (see Figure 3), and

$$
c\left(x_{0}\right)=n \text {-dimensional volume of } \Omega \text {. }
$$

LEMMA 4.5. With the above notation

$$
\operatorname{volume}\left(\operatorname{ch} S_{t x_{0}}\left(K^{*}\right)\right) \approx \frac{\left|\eta_{0}\right|}{\sqrt{1-t^{2}}} \cdot \operatorname{vol}(\Omega)
$$

holds as $t \rightarrow 1$. 


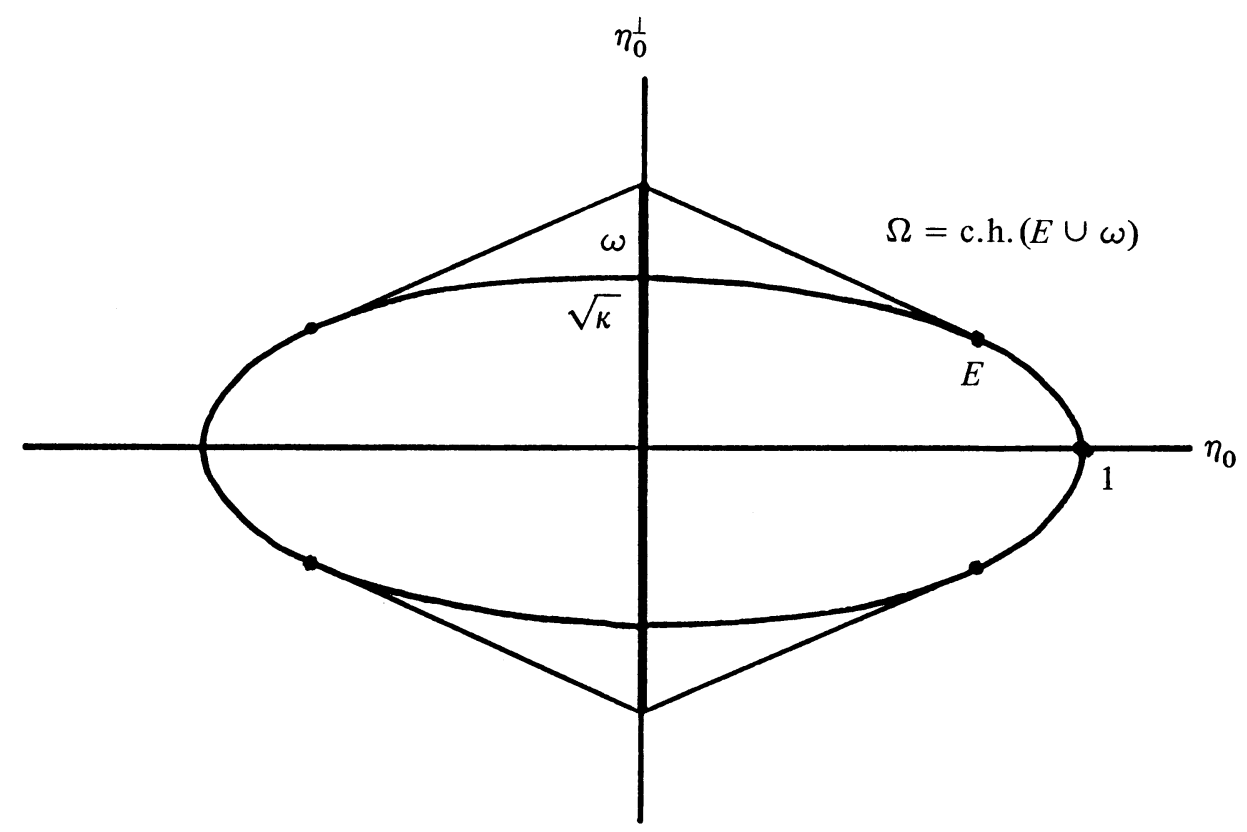

FIGURE 3

PROOF. We will show that

$$
\lim _{t \rightarrow 1} W_{t}\left(\operatorname{ch} S_{t x_{0}}\left(K^{*}\right)\right)=\operatorname{ch}\left(\omega \cup E_{1}\right) .
$$

It follows, then, that the volume of $\operatorname{ch} S_{t x_{0}}\left(K^{*}\right)$ is $\left(1-t^{2}\right)^{-1 / 2}$ times

$$
\left|\eta_{0}\right| \cdot \operatorname{vol}\left(\operatorname{ch}\left(\omega \cup E_{1}\right)\right) \text {, }
$$

where the factor $\left|\eta_{0}\right|$ enters because $\left|\eta_{0}\right|$ is the unit of length on the $\eta_{0}$-axis. Thus, the lemma follows from (4.4).

To show (4.4), we use the notation $\omega_{t}=\left(\eta_{0}^{\perp}\right) \cap S_{t x_{0}}\left(K^{*}\right)$. From Lemma 4.3 we have

$$
W_{t}\left(\operatorname{ch} S_{t x_{0}}\left(K^{*}\right) \cap\left(\eta_{0}^{\perp}\right)\right) \supset \omega_{t}
$$

and so

$$
\lim _{t \rightarrow 1} W_{t}\left(\operatorname{ch} S_{t x_{0}}\left(K^{*}\right) \cap\left(\eta_{0}^{\perp}\right)\right) \supset \operatorname{ch}\left(\omega_{t} \cup E\right)
$$

Thus, $\supset$ holds in (4.4).

For the reverse inclusion, we note that for any $\varepsilon>0$,

$$
\begin{aligned}
S_{t x_{0}}\left(K^{*}\right) \subset S_{x_{0}}\left(K^{*} \cap\{\mid \eta\right. & \left.\left.-\eta_{0} \mid>\varepsilon\right\}\right) \cup S_{t x_{0}}\left(K^{*} \cap\left\{\left|\eta-\eta_{0}\right|>\varepsilon\right\}\right) \\
& =\bar{K}_{1} \cup \bar{K}_{2}(t) .
\end{aligned}
$$

Thus,

$$
\begin{aligned}
\lim _{t \rightarrow 1} W_{t}\left(\operatorname{ch} S_{t x_{0}}\left(K^{*}\right)\right) & \subset \lim _{t \rightarrow 1} W_{t}\left(\operatorname{ch}\left(\bar{K}_{1} \cup \bar{K}_{2}(t)\right)\right. \\
& =\lim _{t \rightarrow 1} \operatorname{ch}\left(W_{t} \bar{K}_{1} \cup W_{t} \bar{K}_{2}(t)\right) \subset \operatorname{ch}(\omega \cup E)=\Omega
\end{aligned}
$$


where the next-to-last inclusion follows from Lemma 4.4.

PROOF OF THEOREM 1.4. We will show that the quantity

$$
c\left(x_{0}\right)=(\operatorname{vol}(\Omega)) / \sqrt{2 \cdot \rho_{K}\left(x_{0}\right)}
$$

is the asymptotic value in Theorem 1.4. First, we note that, with the notation of Theorem 1.4, $|x-\bar{x}|=\operatorname{dist}(x, \partial K)$ and for fixed $x_{0} \in \partial K$

$$
\operatorname{dist}\left(t x_{0}, \partial K\right) \approx|1-t| x_{0} \cdot \frac{\eta_{0}}{\left|\eta_{0}\right|}=\frac{1-t}{\left|\eta_{0}\right|}
$$

holds for $t \rightarrow 1$. Now by Lemma 4.5 we have, with $\Omega=\Omega_{x_{0}}$,

$$
\begin{aligned}
\lambda\left(t x_{0}\right) & =\operatorname{vol} \operatorname{ch} S_{t x_{0}}\left(K^{*}\right) \approx \frac{\left|\eta_{0}\right| \operatorname{vol}(\Omega)}{\sqrt{1-t^{2}}} \approx \frac{\left|\eta_{0}\right| \operatorname{vol}(\Omega)}{\sqrt{2} \sqrt{1-t}}=\frac{\left|\eta_{0}\right|^{1 / 2} \operatorname{vol}(\Omega)}{\sqrt{2}\left(\operatorname{dist}\left(t x_{0}, \partial K\right)\right)^{1 / 2}} \\
& =\frac{\operatorname{vol}\left(\Omega_{x_{0}}\right)}{\left(2 \cdot \rho_{K}\left(x_{0}\right)\left|t x_{0}-\bar{x}\right|\right)^{1 / 2}} \approx \frac{\operatorname{vol}\left(\Omega_{x_{0}}\right)}{\left(2 \cdot \rho_{K}(\bar{x}) \cdot\left|t x_{0}-\bar{x}\right|\right)^{1 / 2}}
\end{aligned}
$$

since $\eta_{0}$ was normalized so that $x_{0} \cdot \eta_{0}=1$; i.e., $\rho_{K}\left(x_{0}\right)=\left|\eta_{0}\right|^{-1}$.

\section{REFERENCES}

1. E. Bedford and B. A. Taylor, The Dirichlet problem for a complex Monge-Ampere equation, Invent. Math. 37 (1976), 1-44.

2. __ A new capacity for plurisubharmonic functions, Acta Math. 149 (1982), 1-40.

3. __ Fine topology, Silov boundary, and $\left(d d^{c}\right)^{n}$, preprint.

4. __ On the convex hull of ellipsoids, preprint.

5. N. Levenberg, Monge-Ampère measures associated to extremal plurisubharmonic functions in $\mathbf{C}^{n}$, Trans. Amer. Math. Soc. 289 (1985), 333-343.

6. M. Lundin, The extremal plurisubharmonic function for convex symmetric subsets of $\mathbf{R}^{n}$, Michigan Math. J. 32 (1985), 197-201.

7. _ An explicit solution to the complex Monge-Ampere equation, preprint.

8. Nguyen Thanh Van and A. Zeriahi, Familles de polynomes presque partout bornées, Bull. Sci. Math. 197 (1983), 81-91.

9. W. Plesniak, Sur la L-regularité des compacts de $\mathbf{C}^{n}$, Séminaire d'Analyse Complex de Toulouse.

10. _ L-regularity of subanalytic sets in $\mathbf{R}^{n}$, preprint.

11. J. Rauch and B. A. Taylor, The Dirichlet problem for the multidimensional MongeAmpere equation, Rocky Mountain J. Math. 7 (1977), 345-364.

12. A. Sadullaev, Plurisubharmonic measures and capacities on complex manifolds, Russian Math. Surveys 36 (1981), 61-119.

13. J. Siciak, Extremal plurisubharmonic functions in $\mathbf{C}^{n}$, Proc. 1st Finnish-Polish Summer School in Complex Analysis, 1977, pp. 115-152.

14. __ On some inequalities for polynomials, Zeszty Nauk. Uniw. Jagielloń 21 (1979), 7-10.

15. __ Extremal plurisubharmonic functions and capacities in $\mathbf{C}^{n}$, Sophia Kokyuroku Math. 14, Sofia Univ., Tokyo, 1982.

16. B. A. Taylor, An estimate for an extremal plurisubharmonic function in $\mathbf{C}^{n}$, Séminaire d'Analyse, P. Lelong-P. Dolbeault-H. Skoda, 1982-1983, Lecture Notes in Math., vol. 1028, Springer, 1983, pp. 318-328.

17. V. Zaharjuta, Orthogonal polynomials, and the Bernstein-Walsh theorem for analytic functions of several complex variables, Ann. Polon. Math. 33 (1976), 137-148. 47405

DEPARTMENT OF MATHEMATICS, INDIANA UNIVERSITY, BLOOMINGTON, INDIANA

Department of Mathematics, University of Michigan, Ann Arbor, Michigan 48109 